\title{
Nueva metodología geométrica para evaluar la morfología del eritrocito normal
}

\author{
New physical and mathematical methodology to evaluate the morphology \\ of normal erythrocyte
}

Javier Rodríguez ${ }^{1}$, Santiago Escobar², Lesly Abder², Johnsen del Río², Luis Quintero², David Ocampo²

\section{Resumen}

Objetivo. Desarrollar una nueva metodología para caracterizar la estructura del eritrocito normal mediante el espacio ocupado por el anillo del eritrocito normal caracterizado con el método de Box Counting. Método. se analizaron las imágenes de 20 extendidos de sangre periférica, cuyos eritrocitos fueron evaluados por un experto como normales. Se superpusieron dos rejillas Kp de 5 x 5 pixeles y $\mathrm{Kg}$ de 10 x 10 pixeles, para calcular el espacio ocupado por dos regiones del eritrocito estos son, el disco y centro de este, visto de manera frontal mediante el método de Box Counting. Resultados. Los espacios ocupados por la región del disco con la rejilla Kp variaron entre 47 y 56, la región del centro del eritrocito, varió entre 9 y 14. La dimensión fractal de estas dos regiones varió entre 0,941 y 1,115 para el disco, entre 0,652 y 1,222 para el centro. Conclusiones. La estructura del eritrocito normal puede ser caracterizada mediante el espacio ocupado por cada una de las regiones del eritrocito a partir de la geometría fractal.

Palabras claves: matemáticas, fractales, hematología, eritrocito, morfofisiología, geometría.

\begin{abstract}
Objective. Develop a new methodology to characterize the structure of the normal erythrocyte through the space occupied by the ring of the normal erythrocyte characterized by the method of Box Counting. Method. Images of 10 peripheral blood smears were analysed, whose erythrocytes were evaluated by an expert as normal. There were superimposed two Kp grids of $5 \times 5$ pixels and $\mathrm{Kg}$ of $10 \times 10$ pixels, to calculate the space occupied by two regions of the erythrocyte which are, disc and centre of this, seen of way frontal by the method of Box Counting. Results. The spaces occupied by the disc region with grid $\mathrm{Kp}$ varied between 47 and 56, the central region of the erythrocyte, varied between 9 and 14 . The fractal dimension of these two regions varied between 0,941 and 1,115 for the disc, between 0.652 and 1,222 for the centre. Conclusions. The normal erythrocyte structure can be characterized by the space occupied by the regions erythrocyte from fractal geometry.
\end{abstract}

Keywords: Mathematics, fractals, hematology, erythrocyte, morphophysiology, geometry.

1. Director Grupo Insight. Centro de Investigaciones - Clínica del Country.

2. Estudiante e Investigador, Grupo Insight, Colegio Benjamín Herrera. 


\section{Introducción}

La geometría euclidiana es un conjunto de propiedades y relaciones geométricas de las figuras en el plano o el espacio. La regularidad de las figuras se caracteriza mediante reglas euclidianas como son longitud, área y volumen. Por el contrario, si una figura o el objeto presenta una forma irregular (1-3), dicha irregularidad puede ser cuantificable mediante la geometría fractal calculando para ello la dimensión fractal (3). El objeto o la figura en esta geometría se denominan fractal. Existen varios tipos de fractales, cada uno de ellos cuenta con un método matemático que permite calcular la dimensión fractal. Entre ellos se encuentra el fractal salvaje, se caracteriza por la superposición de sus partes y su irregularidad es calculada mediante el método de Box-Counting (4). Con este método se puede calcular la distribución espacial de un objeto particular, observado a diferentes escalas mediante la superposición de rejillas con distinta dimensión.

En hematología, el extendido de sangre periférica se lleva a cabo en los casos en que los hemogramas presentan alguna desviación en los recuentos directos, indirectos calculados, también en los casos de presentarse una enfermedad con y sin origen hematológico definido. Mediante este procedimiento se puede observar otras características como son alteraciones morfológicas de los eritrocitos, leucocitos y plaquetas (5). Estas alteraciones son observadas a través de microscopio de luz, la muestra de sangre periférica es coloreada por dos tinciones la de Wright y Romanowsky. El eritrocito normal visto de manera lateral presenta una forma bicóncava, al verlo de manera frontal tiene forma de disco cóncavo. Adicionalmente, se le atribuye una redondez cuyo diámetro varía entre $7 \mu \mathrm{m}$ y $9 \mu \mathrm{m}$. $\mathrm{Al}$ presentarse una variación en las características anteriormente mencionadas, se debe considerar la muestra como anormal, la cual puede estar posiblemente asociada a una enfermedad (5).

Existen alteraciones que al ser observadas en las muestras a través del microscopio, requieren im- plementar otros métodos desarrollados en el contexto molecular, los cuales pueden determinar anomalías genéticas. Estos métodos también son utilizados para evaluar la respuesta a terapias y el diagnóstico de enfermedades comunes como son el VIH/SIDA, la malaria y la tuberculosis (6). Sin embargo, debido a los altos costos que representa este tipo de diagnósticos (6), se hace necesario que los especialistas y practicantes de laboratorio se formen para realizar un correcto análisis morfológico. Entre los estudios desarrollados en sujetos infectados por malaria, Pinzón y colaboradores, desarrollaron una técnica de conteo de glóbulos rojos, que reduce el tiempo de la lectura de las láminas, al diferenciar según la forma los eritrocitos los sujetos infectados con malaria (7).

A la luz de estos resultados se han desarrollado nuevas metodologías con el fin de caracterizar las alteraciones de los eritrocitos mediante en el contexto de teorías físicas, matemáticas y geométricas como son la geometría fractal y euclidiana (8-10). La primera metodología reveló que la aparente regularidad de un eritrocito normal y las alteraciones de un eritrocito cuando este se sale de los parámetros normales puede ser cuantificada indistintamente de las clasificaciones de laboratorio clínico, mediante la geometría fractal (8). Esta metodología al ser aplicada para evaluar otras muestras de extendido de sangre periférica sirvió de base para la creación de una metodología diagnóstica, que de manera simultánea evalúa las alteraciones de la estructura de los eritrocitos mediante la geometría euclidiana y fractal. La metodología evidenció una relación entre las proporciones de la superficie y borde de la estructura del eritrocito, permitiendo diferenciar de manera objetiva el eritrocito normal respecto a las distintas alteraciones de este (9). Posteriormente, con la metodología mencionada se realizó una simulación teórica que permite detallar la evolución de las alteraciones morfológicas del eritrocito, además de facilitar su seguimiento en el tiempo (10). 
El propósito de la presente investigación es aplicar las metodologías previamente desarrolladas para realizar una nueva caracterización de las dos regiones del eritrocito normal el disco y centro de este, en el contexto de la geometría fractal mediante el método de Box Counting $(8,9)$.

\section{Materiales y métodos}

Método de Box Counting: es una medida matemática adimensional con la cual es posible cuantificar el grado de irregularidad de un objeto; siendo K: el grado de partición de la cuadrícula, N: es el número de espacios que contiene el contorno del objeto y D: la dimensión fractal.

$$
D=\frac{\log N\left(2^{-(K+1)}\right)-\log N\left(2^{-K}\right)}{\log 2^{k+1}-\log 2^{k}}=\log _{2} \frac{N\left(2^{-(k+1)}\right)}{N\left(2^{-k}\right)}
$$

Regiones: la estructura de un eritrocito normal que al ser vista de manera frontal, tiene forma de un disco cóncavo. La región uno (R1) representa el disco y la región dos (R2) corresponde al centro de este.

Rejillas: consiste en una cuadrícula con dimensiones de 5x5 y 10x10 pixeles. En donde la cuadrícula de $5 \times 5$ pixeles, se denomina rejilla de cuadros pequeños $(\mathrm{Kp})$ y la cuadrícula de $10 \times 10$ pixeles, rejilla de cuadros grandes.

Superficie en pixeles de las regiones uno y dos: número de pixeles que tocan el interior o el borde de la imagen medida.

\section{Procedimiento}

Se seleccionaron diferentes tipos de muestras de sangre, del Laboratorio de referencia en Morfología Hematológica. Los eritrocitos normales fueron evaluados por el especialista del laboratorio según los parámetros convencionales. Las mues- tras de sangre fueron observadas en extendidos tratados con tinte de Wright, con un aumento de $10 x$ por $100 x$ a través de un microscopio Carl Zeiss y las imágenes fueron tomadas por una Cámara de microscopio digital.

Las imágenes de los eritrocitos normales seleccionados para el estudio fueron editadas en Microsoft Office Professional Plus 2010, para dejarlas en una dimensión de 448 x 336 pixeles para que fueran reconocidas por el software previamente desarrollado por el grupo Insight. Posteriormente, fueron delineadas las dos regiones de 20 eritrocitos normales, tal y como se puede ver en la figura $1 \mathrm{~b}$ y $1 \mathrm{c}$. Posteriormente, se superpusieron las dos rejillas en cada una de las regiones, la rejilla Kp superpuesta en las dos regiones se puede observar en la figura $1 \mathrm{~d}$ y $1 \mathrm{c}$, para evaluar mediante el método de Box Counting cuántos espacios ocupa las dos regiones de los eritrocitos normales, así como su dimensión fractal. De las dos rejillas utilizadas, los valores que permiten caracterizar con más detalle la irregularidad de los eritrocitos normales es la rejilla $\mathrm{Kp}$, los valores de la rejilla $\mathrm{Kg}$, no fueron consideradas en esta investigación, sino para el cálculo de la dimensión fractal. Finalmente, se calculó las proporciones entre la superficie y la rejilla $\mathrm{Kp}$, para establecer valores que sean útiles para comparar y diferenciar entre otras regiones de eritrocitos normales.

\section{Resultados}

La imagen de uno de los eritrocitos seleccionado para evaluar las dos regiones R1 y R2, se pueden observar en la Figura 1 y 2. Los valores ocupados por la R1 calculados con la rejilla Kp variaron entre 47 y 56. La R2 con la rejilla $\mathrm{Kg}$ la superficie varió entre 9 y 14 . La dimensión fractal para la R1 vario entre 0,94 y 1,12 , para la R2 la dimensión fractal varió entre 0,65 y 1,22. La superficie de la R1 vario entre 1181 y 1561, la R2 varió entre 82 y 138. La proporción entre la superficie y la rejilla Kp varió entre 22,79 y 32,25, Tabla 1 . 
Tabla 1. Medidas de la región uno (R1) y dos (R2) de 10 de los 20 extendidos de sangre periférica de eritrocitos normales. Kp: es en número de cuadros ocupados por R1 y R2 con la rejilla de 5 x 5 pixeles. Df: es la dimensión fractal; S: superficie. S/Kp: proporción entre la superficie y el número ocupados con la rejilla Kp.

\begin{tabular}{|l|l|l|l|l|l|l|l|l|l|}
\hline & \multicolumn{1}{|l|}{$\mathbf{R 1}$} & \multicolumn{1}{|l|}{$\mathbf{R 2}$} \\
\hline No & Kp & Kg & Df & S & S/Kp & Kp & Df & S & S/Kp \\
\hline 1 & 47 & 24 & 0,97 & 1181 & 25,13 & 10 & 0,74 & 105 & 10,50 \\
\hline 2 & 48 & 25 & 0,94 & 1548 & 32,25 & 9 & 0,85 & 82 & 9,11 \\
\hline 3 & 49 & 25 & 0,97 & 1199 & 24,47 & 11 & 0,65 & 155 & 14,09 \\
\hline 4 & 50 & 24 & 1,06 & 1411 & 28,22 & 11 & 1,14 & 130 & 11,82 \\
\hline 5 & 51 & 26 & 0,97 & 1521 & 29,82 & 10 & 0,74 & 150 & 15,00 \\
\hline 6 & 52 & 24 & 1,12 & 1250 & 24,04 & 14 & 0,81 & 180 & 12,86 \\
\hline 7 & 53 & 25 & 1,08 & 1208 & 22,79 & 13 & 0,89 & 204 & 15,69 \\
\hline 8 & 54 & 28 & 0,95 & 1415 & 26,20 & 10 & 0,74 & 127 & 12,70 \\
\hline 9 & 55 & 26 & 1,08 & 1561 & 28,38 & 13 & 1,12 & 238 & 18,31 \\
\hline 10 & 56 & 27 & 1,05 & 1511 & 26,98 & 14 & 1,22 & 203 & 14,50 \\
\hline
\end{tabular}

Fuente: Elaborada por los autores.

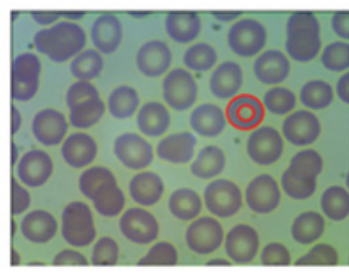

a

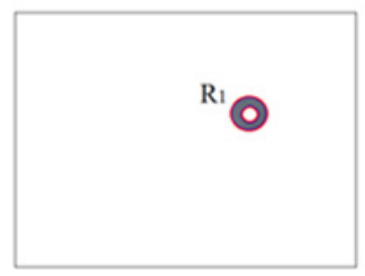

b

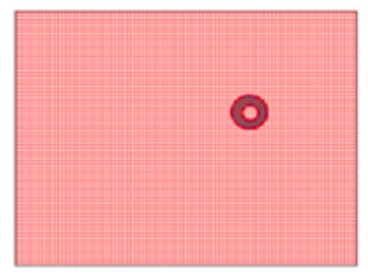

c

Figura 1. Imagen de extendido de sangre periférica con eritrocitos normales. a) Eritrocito normal delineado para ser evaluado; b) Región uno (R1) del eritrocito normal delineado y separado del extendido; c) R1 al que fue superpuesta la rejilla Kp de 5 x 5 pixeles.

Fuente: Elaborada por los autores.

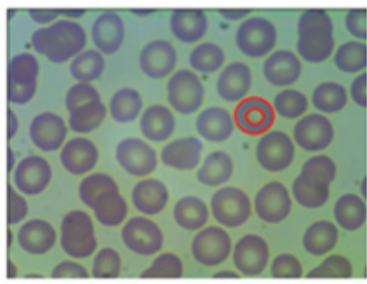

a

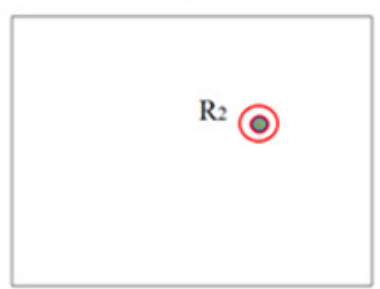

b

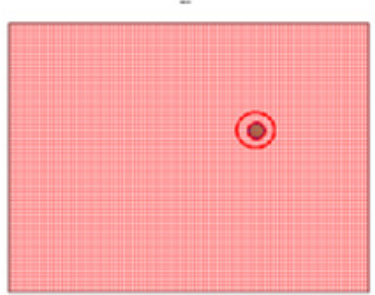

c

Figura 2. Imagen de extendido de sangre periférica con eritrocitos normales. a) Eritrocito normal delineado para ser evaluado; b) Región dos (R2) del eritrocito normal delineado; c) separación de la R2 al que fue superpuesta la rejilla Kp de 5 x 5 pixeles.

Fuente: Elaborada por los autores. 


\section{Discusión}

Este es el primer trabajo en el que se desarrolla una metodología geométrica para caracterizar el espacio ocupado por el disco y el centro del eritrocito normal, en el contexto de la geometría fractal y las medidas establecidas en trabajos previamente desarrollados. La superposición de la rejilla Kp permitió caracterizar el rango de valores normales matemáticos en los cuales puede variar el espacio ocupado por el disco y el centro de cada eritrocito. Este método contribuye a realizar medidas de forma más precisa, en particular en aquellas alteraciones que estén indicando indicios de alguna patología.

La presente investigación evidencia un nuevo sistema de medida que contribuye en la comprensión de la estructura del eritrocito cuando este es normal. En el futuro la metodología será aplicada para caracterizar otras posibles alteraciones de los eritrocitos normales de manera independiente a las causas que den origen a dichas alteraciones. Más aún, este nuevo sistema de medida puede ser útil para evaluar el cambio entre la configuración y las propiedades mecánicas de los eritrocitos afectados por la radiación (11). Cambios que al ser analizados desde la noción de superficie, clínicamente son considerados como causa de obstrucción en la circulación (11).

Las metodologías previamente desarrolladas para evaluar las estructuras eritrocitarias normales y anormales, y la metodología desarrollada en el presente trabajo, evidencian lo adecuado que resulta caracterizar estas estructuras desde la noción de la geometría fractal, logrando como resultado medidas objetivas y reproducibles independientes de las causas que desde el ámbito clínico se atribuyen a las alteraciones de los eritrocitos $(8,9)$. En un estudio recientemente se establecieron las posibles trayectorias de las alteraciones de los eritrocitos partiendo de los valores de las dimensiones de los eritrocitos normales y equinocitos hallados previamente, mediante la aplicación simultánea de la geometría fractal y euclidiana (10). La metodología mostró cómo ambas geometrías pueden caracterizar la estructura eritrocitaria, mediante las nociones de irregularidad, área, la superficie y las regiones. Adicionalmente, permite estudiar estas alteraciones como un fenómeno finito que facilita el seguimiento en el tiempo de estas alteraciones que sirvan como indicadores tempranos del progreso de pacientes que presentan equinocitos en el hemograma (10).

Por muchos años se viene analizando la estructura de los eritrocitos y sus cambios desde las nociones de la geometría euclidiana, relacionando los eritrocitos con figuras que tienen por forma un anillo o disco cóncavo, esfera y elipse $(5,7,11)$. A partir de estas nociones se han realizado la clasificación de los eritrocitos, las cuales resultan a menudo insuficientes para hacer frente a ciertos problemas, tales como la variación de un eritrocito cuando está comenzando la infección de la malaria (12) u otra patología; así como para evaluar la efectividad y adversidades de un tratamiento terapéutico (13), sea este por radiación de rayos $\mathrm{X}$ (14). En cambio, estudios realizados desde el contexto de la geometría fractal han evidenciado que un comportamiento constante de la interacción entre los eritrocitos y las macromoléculas, se puede evidenciar mediante medidas fractales (15). Por otro lado, los valores de la dimensión fractal de los agregados de sedimentación, generan un modelo matemático que permite predecir su comportamiento (16).

En consecuencia, el presente estudio cuantifica las características morfológicas del eritrocito, desde el espacio ocupado por ambas regiones de forma análoga a como se hecho con otras estructuras como son arterial, ventricular y en células de cuello uterino (17-19). Más aún, el fenómeno de alteración eritrocitaria es observado de forma no continua sino discreta. Lo anterior se deduce de la noción de ventana temporal establecida por Prigogine (20), que da cuenta de fenómenos acausales cuya finalidad se centra en el estable- 
cimiento de órdenes matemáticos subyacentes a la estructura del eritrocito normal independientemente de cualquier análisis poblacional, siendo posible contrastarlos en cada caso particular. Otro estudio que sirve de ejemplo de esta noción se puede encontrar en la metodología diagnóstica desarrollada para evaluar los grados de alteración de las células de cuello uterino (21). Como resultado de la aplicación y refinamiento de esta metodología en varias investigaciones, se estableció una generalización en la cual se puede observar todas las posibles trayectorias de evolución de las células de cuello uterino desde normalidad hasta neoplasia (22).

Como resultado de la aplicación de teorías físicas, matemáticas y geométricas en el desarrollo de metodologías de aplicación clínica se ha proporcionado soluciones en otros ámbitos de la medicina como son en el estudio de la dinámica cardiaca de adulto (23), fetal (24), y neonatal (25), logrando recientemente una metodología que predice casos de mortalidad (26). En epidemiología, se han desarrollado predicciones de brotes de malaria en 820 municipios de Colombia en rangos de tres semanas, con un 99,86\% de efectividad (27). También se han desarrollado métodos predictivos en inmunología (28), biología molecular (29) e infectología (30-33). Estas investigaciones permiten evidenciar la aplicabilidad práctica que tiene el uso de las mismas en las diferentes ramas de la medicina.

\section{Agradecimientos}

Agradecemos a Yolanda Soracipa por la colaboración en la redacción del manuscrito.

Extendemos nuestro agradecimiento al Centro de Investigaciones de la Clínica del Country, a los Doctores Tito Tulio Roa, Director de Educación Médica; Jorge Ospina, Director Médico; Alfonso Correa, Director del Centro de Investigaciones, y a las Doctoras Adriana Lizbeth, epidemióloga y Silvia Ortiz, Enfermera Jefe del Centro de Inves- tigaciones, por su apoyo durante ańos a nuestras investigaciones. A las directivas del Colegio Técnico Benjamín Herrera, por el apoyo brindado a la propuesta de formar estudiantes de secundaria en el campo científico. En especial al Déiber Villar, Rector, del Colegio Técnico Benjamín Herrera; a Leonardo Quintero, Docente de Química; y Gonzalo Vanegas, Docente de Física, por abrir espacios académicos para la ejecución de la propuesta.

\section{Dedicatoria}

A los hijos del primer autor.

\section{Referencias}

1. Mandelbrot B. How Long Is the Coast of Britain? Statistical Self-Similarity and Fractional Dimension. Science, 1967; 156: 636-38.

2. Mandelbrot B. Árboles jerárquicos o de clasificación, y la dimensión. En: Los Objetos Fractales. Barcelona: Tusquets Eds S.A. 2000: 161-6.

3. Mandelbrot B. The Fractal Geometry of Nature. San Francisco: Freeman Ed; 1972. p. 341-348.

4. Peitgen H, Jürgens H, Saupe D. The Box-Counting Dimension En: Chaos and Chaos and Fractals: New Frontiers of Science. New York: Springer-Verlag. 1992. pp.

5. Campuzano G. Utilidad clínica del extendido de sangre periférica: los eritrocitos. Medicina \& Laboratorio. 2008; 14(7-8): 311-313. Disponible en: http://www.medigraphic.com/pdfs/ medlab/myl-2008/myl087-8b.pdf.

6. Alice Charwudzi, Edeghonghon E. Olayemi, Ivy Ekem, Olufunmilayo Olopade, Mariann Coyle, Amma Anima Benneh, Emmanuel Alote Allotey. A Preliminary Study of the Suitability of Archival Bone Marrow and Peripheral Blood Smears for Diagnosis of CML Using FISH. Adv Hematol. 2014; 2014: 604165 .

7. Pinzón R, Garavito G, Hata Y, Artega L, García J.D. Desarrollo de un sistema de análisis automático de imágenes de Extendido Sanguíneos. En Memorias del Congreso Español de la Sociedad de Ingeniería Biomédica; 2004. p.45-59.

8. Rodríguez J, Correa C, Prieto S, Ospino B, Bernal P, Ortiz L, Munévar A. Caracterización geométrica de los glóbulos rojos. Diferenciación de muestras normales y patológicas. Rev. Cienc. Salud. 6 (3): 47-56.

9. Correa C, Rodríguez J, Prieto S, Álvarez L, Ospino B, Munévar A, et al. Geometric diagnosis of erythrocyte morphophysiology. J. Med. Med. Sci. 2012; 3(11): 715-720. 
10. Rodríguez J. Prieto S, Correa S, Mejía M, Ospino B, Munevar Á, Amaya B, Duarte Y, Medina S, Felipe C. Simulación de estructuras eritrocitarias con base en la geometría fractal y euclidiana. Arch Med (Manizales) 2014, 14(2): 276-284.

11. Monica Diez-Silva, Ming Dao, Jongyoon Han, Chwee-Teck Lim, Subra Suresh. Shape and Biomechanical Characteristics of Human Red Blood Cells in Health and Disease. MRS Bull. 2010 May; 35(5): 382-388.

12. Thurston Herricks,a Karl B. Seydel, Malcolm Molyneux, Terrie Taylor, and Pradipsinh K. Rathoda. Estimating Physical Splenic Filtration of Plasmodium falciparum Infected Red Blood Cells in Malaria Patients. Cell Microbiol. 2012 Dec; 14(12): 1880-1891.

13. Zuk A, Targosz M, Szymonski M. Effect of selected drugs used in asthma treatment on morphology and elastic properties of red blood cells. Int J Nanomedicine. 2011; 6: 249-257.

14. Xu D, Peng M, Zhang Z, Dong G, Zhang Y, Yu H. Study of

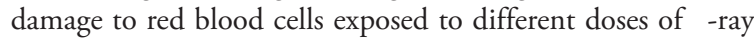
irradiation. Blood Transfus. 2012; 10(3): 321-330.

15. Kuo CD, Bai JJ, Chien S. A fractal model for erythrocyte sedimentation. Biorheology. 1994; 1:77-89.

16. Tang P, Greenwood J, Raper JA. A model to describe the settling behavior of fractal aggregates. J Colloid Interface Sci. 2002; 247(1):210-9.

17. Rodriguez J, Prieto S, Correa C, Bernal P, Puerta G, Vitery $S$, et al. Theoretical generalization of normal and sick coronary arteries with fractal dimensions and the arterial intrinsic mathematical harmony. BMC Medical Physics. 2010; 10:1-6.

18. Rodríguez J, Prieto S, Correa C, Bernal P, Álvarez L, Forero G, et al. Diagnóstico fractal del ventriculograma cardiaco izquierdo. Geometría fractal del ventriculograma durante la dinámica cardiaca. Rev Colomb Cardiol. 2012; 19(1):18-24.

19. Velásquez J, Prieto S, Catalina C, Dominguez D, Cardona DM, Melo M. Geometrical nuclear diagnosis and total paths of cervical cell evolution from normality to cancer. Journal of Cancer Research and Therapeutics 2015; 11(Issue 1): 98-104.

20. Fernández A. Orden y Caos. Introducción. En: Ed. Prensa Científica S.A. España; 1990; p.4-8.

21. Prieto S, Rodríguez J, Correa C, Soracipa Y. Diagnosis of cervical cells based on fractal and Euclidian geometrical measurements: Intrinsic Geometric Cellular Organization. BMC Medical Physics 2014, 14(2):1-9.

22. Velásquez J, Prieto S, Catalina C, Dominguez D, Cardona DM, Melo M. Geometrical nuclear diagnosis and total paths of cervical cell evolution from normality to cancer. Journal of Cancer Research and Therapeutics 2015; 11 (Issue 1): 98-104.

23. Rodríguez J, Prieto S, Domínguez D, Melo M, Mendoza F, Correa C, et al. Mathematical-physical prediction of cardiac dynamics using the proportional entropy of dynamic systems. J. Med. Med. Sci. 2013; 4(8):370-381

24. Rodríguez J. Nuevo diagnóstico físico y matemático de la monitoria fetal: predicción de aplicación clínica. Momento Revista de Física. 2012; 44: 49-65.
25. Rodríguez J, Prieto S, Flórez M, Alarcón C, López R, Aguirre $\mathrm{G}$, et al. Physical-mathematical diagnosis of cardiac dynamic on neonatal sepsis: predictions of clinical application. J. Med. Med. Sci.2014; 5(5): 102-108.

26. Rodríguez J. Dynamical systems applied to dynamic variables of patients from the intensive care unit (ICU): Physical and mathematical mortality predictions on ICU. J. Med. Med. Sci. 2015; 6(8):209-220.

27. Rodríguez J. Método para la predicción de la dinámica temporal de la malaria en los municipios de Colombia. Rev Panam Salud Pública. 2010; 27(3):211-8.

28. Rodríguez J, Bernal P, Álvarez L, Pabón S, Ibáñez S, Chapuel $\mathrm{N}$, et al. Predicción de unión de péptidos de MSP-1 y EBA140 de plasmodium falciparum al HLA clase II Probabilidad, combinatoria y entropía aplicadas a secuencias peptídicas. Inmunología. 2010; 29(3):91-99.

29. Rodríguez J, Bernal P, Prieto S, Correa C. Teoría de péptidos de alta unión de malaria al glóbulo rojo. Predicciones teóricas de nuevos péptidos de unión y mutaciones teóricas predictivas de aminoácidos críticos. Inmunología 2010; 29(1):7-19.

30. Rodríguez J, Prieto S, Correa C, Forero M, Pérez C, Soracipa $\mathrm{Y}$, et al. Teoría de conjuntos aplicada al recuento de linfocitos y leucocitos: predicción de linfocitos T CD4 de pacientes con VIH/SIDA. Inmunología. 2013; 32(2): 50-56.

31. Rodríguez J, Prieto S, Correa C, Mora J, Bravo J, Soracipa Y, et al. Predictions of CD4 lymphocytes' count in HIV patients from complete blood count. BMC Medical Physics. BMC Medical Physics. 2013; 13:3.

32. Rodríguez Z, Veloza L, Baena del Valle J, Ramos R, Gómez C, Gómez D. Parámetros hematológicos de pacientes con fibrósis quística de la costa caribe colombiana: análisis y asociación con aislamiento de pseudomonas aerugisona. NOVA. 2009; (7) $11: 91-102$.

33. Páez, L. C. C., et al. Comparación del cultivo celular de HeLa y HEp-2: Perspectivas de estudios con Chlamydia trachomatis. 2015; Nova 13(23) 